Gynäkol Geburtshilfliche Rundsch 1997;37:231-232

\title{
Mitteilungen aus Österreich
}

Veranstaltungen der Osterreichischen Gesellschaft fiir Gynäkologie und Geburtshilfe (ÖGGG)

Weitere Veranstaltungen

\subsection{6.-13.06.1998: Bad Gastein}

Jahrestagung und Fortbildungsveranstaltung der Osterreichischen Gesellschaft fiir Gynäkologie und Geburtshilfe

Kontaktadresse: Prim. Prof. Dr. H. Salzer Vorstand der gynäkologischgeburtshilflichen Abteilung Wilhelminenspital, Montleartstrasse 37, A-1 160 Wien Tel. +431491504701, Fax +431491504709

Sitzungen der Osterreichischen Gesellschaft fiir Prä- und Perinatale Medizin 13.06.1998: Baden/Wien 17.10.1998: Feldkirch 05.12.1998: St. Pölten 15.10-17.10.1998: Melk Aktueller Stand der Therapie der grnäkologischen Malignome 'State of the Art' Kontaktadresse: Prim. Prof. Dr. H. Salzer Vorstand der gynäkologischgeburtshilflichen Abteilung Wilhelminenspital, Montleartstrasse 37, A-1 160 Wien Tel. +43149150 4701, Fax +431491504709

Sitzung der Osterreichischen Gesellschaft zum Studium der Sterilität und Fertilität 10.11.1998: Wien 30.05.-05.06.1998: Korfu (Griechenland)

V. Mediterranes Fortbildungsseminar: Ultraschall in Geburtshilfe und Gynäkologie ('Sun and Sound')

Kontaktadresse: Prof. Dr. G. Bernaschek und Prof. Dr. J. Deutinger Institut Gynschall, Theresiengasse 46, A-1 180 Wien Tel. +43 140282 24, Fax +43 140212 32

18.06.-20.06.1998: Hof/Salzburg

6. Symposium «Der medizinische Videofilm»

Kontaktadresse: EMC - Eva Maier-Congress

Postfach46, A-1095Wien

Tel. +431 4080059, Fax +4314022030

20.06.1998: Hotel Martinspark, Dornbirn 4. Frühsommerliches Symposium der Abteilung fiir Gynäkologie und Geburtshilfe des Krankenhauses Dornbirn: Pränatale Diagnostik und Therapie

Kontaktadresse: Prim. Univ. Doz. Dr. Walter Neunteufel und Dr. Claudia Johler, Krankenhaus Dornbirn Lustenauerstr. 4, A-6850 Dornbirn Tel. +43 5572303 0, Fax +435572303109 02.07.-04.07.1998: Innsbruck

11th Congress of the International Perinatal Doppler 


\section{Society (IPDS 1998)}

Kontaktadresse: Prof. Dr. Ch. Brezinka Univ.-Frauenklinik, Anichstrasse 35, A-6020

Innsbruck Tel. +43 512504 3135, Fax +43 512293967

08.10.-10.10.1998: Graz

Jahrestagung der Osterreichischen Gesellschaft fiir

Reproduktionsmedizin und Endokrinologie

Kontaktadresse: Prof. Dr. W. Urdl

Geburtshilflich-gynäkologischeUniversitäts-Klinik

Auenbruggerplatz, A-8036 Graz

Tel. +43 316385 2201, Fax +433163853061

05.11.-07.11.1998: Innsbruck

Österreichischer Senologiekongress 1998

15. Jahrestagung der Osterreichischen Gesellschaft

fiir Senologie

Kontaktadresse: Dr. P. Springer Universitäts-Klinik für Radiodiagnostik

Anichstrasse 35, A-6020 Innsbruck Tel.+43 512504 2761, Fax+43 5125042758

26.11.-28.11.1998: Wien

«Ultraschall-aktuell» - Ultraschall in Geburtshilfe und

Gynäkologie

Kontaktadresse: Prof. Dr. G. Bernaschek und Prof. Dr. J. Deutinger Gynschall, Theresiengasse 46, A-1 180 Wien Tel. +43 1 4028424, Fax +431 4021232

\section{KAHGEK}

Fax+4161306 1234 E-Mail karger@karger.ch www.karger.com

(C) 1998 S. Karger AG, Basel

This article is also accessible online at: http://BioMedNet.com/karger

231

Ausschreibungen

Hofrat-Erwin-Domanig-Stipendium

\section{Anton-von-Eiselsberg-Preis}

Der von der Van-Swieten-Gesellschaft gestiftete Anton-von-Eiselsberg-Preis beträgt für das laufende Jahr ATS 100.000.- und wird hiermit ausgeschrieben. Er ist in erster Linie für junge Kollegin-nen und Kollegen gedacht, da der Preis der Förderung des wissen-schaftlichen Nachwuchses dienen soil.

Österreichische Ärzte sind berechtigt, Arbeiten einzureichen. Die Arbeiten sollen die Ergebnisse eigener wissenschaftlicher, experi-menteller oder klinischer Untersuchungen aus einem Fachgebiet der Medizin zum Gegenstand haben.

Bei Arbeiten, die nur im Manuskript vorliegen, muss der Nach-weis erbracht werden, dass sie von einer Fachzeitschrift zur Publika-tion angenommen worden sind. Bereits publizierte Arbeiten können nur angenommen werden, wenn sie nach dem 30. April 1997 erschie-nen sind.

Habilitationsschriften, sowie Arbeiten, die schon für eine andere Preisausschreibung eingereicht wurden, sind von der Einreichung ausgeschlossen.

Der eingereichten Arbeit (Original mit 5 Kopien) ist eine Erklä-rung des Autors beizuschliessen, dass alle an dem Zustandekommen der Arbeit beteiligten 
wissenschaftlichen Mitarbeiter (einschliesslich Biologen, Chemiker, Psychologen, Statistiker usw.) im Titel oder in Fussnoten oder sonst in irgendeiner Weise genannt sind. Klinik- und Institutsvorstände bzw. Primarärzte oder Leiter von Abteilungen (Departments) werden ersucht, selbst keine Arbeiten einzureichen.

Wissenschaftlichen Arbeiten, welche nicht in deutscher, sondern in englischer Sprache erschienen sind, muss eine deutsche Zusammen-fassung beigefügt werden. Die Einreichung hat bis spätestens 30. Mai 1998 (Datum des Poststempels) an den Sekretär der Van-Swieten-Gesellschaft, p.A. Wr. Med. Akademie, z. Hd. Fr. Silvia Konstantinou, Alserstrasse 4, A-1090 Wien, zu erfolgen.

Förderungspreis für Senologie

Die Österreichische Gesellschaft für Senologie schreibt den Förderungspreis für Senologie in Höhe von ATS 60.000,- für hervorra-gende klinische oder theoretische Arbeiten auf dem Gebiet der Senologie aus. Für diesen Preis können Arbeiten, die innerhalb der Jahre 1997 oder 1998 publiziert bzw. nachweislich zum Druck angenommen worden sind, eingereicht werden. Erstautoren, die im Jahr 1998 das 40. Lebensjahr vollenden oder vollendet haben, sind von einer Bewerbung ausgeschlossen. Der Erstautor gilt als Einreicher.

Bei Einlangen mehrerer förderungswürdiger Arbeiten kann dieser Preis auch auf mehrere Arbeiten (maximal 3) aufgeteilt werden. Die Preisträger verpflichten sich, den Inhalt ihrer Arbeiten auf der näch-sten Jahrestagung vom 5.-7. November 1998 in Innsbruck in Kurz-form zu präsentieren.

Bewerbungen sind bis spätestens 15. Juni 1998 in4facher Ausfer-tigung an den Sekretär der Gesellschaft, Univ.-Prof. Dr. Ch. Dadak, Österreichische Gesellschaft für Senologie, Universitätsfrauenkli-nik für Frauenheilkunde, Währinger Gürtel 1820, A-1090 Wien, Tel. +43 140400 5615, Fax +431404005635 zu richten.

Die Van-Swieten-Gesellschaft, Verein Österreichischer Ärzte, vergibt nach einem jährlich zu fassenden Vorstandsbeschluss, unter Berücksichtigung der jeweiligen finanziellen Gegebenheiten nach entsprechender Ausschreibung ein Stipendium, das nach dem Mitbe-gründer und Ehrenpräsidenten der Gesellschaft, Hofrat Erwin Domanig, benannt ist.

Dieses Stipendium soil in erster Linie dem Kennenlernen und der praktischen Aneignung von neuen Methoden in der medizinischen Diagnostik und Therapie im Rahmen eines Auslandsaufenthaltes dienen; es darf nur an Nachwuchskräfte (Einzelpersonen) verliehen werden, die nach Werdegang und wissenschaftlicher Eignung die Gewähr dafür bieten, dass ein im Interesse des medizinischen Fortschrittes in Österreich fruchtbares Ergebnis zu erwarten ist.

Die Ausschreibung erfolgt nach der 1. Vorstandssitzung des jeweiligen

Präsidentenjahres durch das Sekretariat.

Die Bewerber richten, entsprechend den Ausschreibungsbedin-gungen, ihr Ansuchen an das Sekretariat der Van-Swieten-Gesellschaft mit exakter Angabe des angestrebten Forschungsziels. Dem Ansuchen sind beizuschliessen:

Curriculum vitae.

Verzeichnis der wissenschaftlichen Publikationen.

Detaillierte Angaben über die mit dem Stipendium verbunde-ne Zielsetzung sowie über Ort, Zeit, Dauer und finanzielle Erforder-nisse.

Empfehlungen des Vorstandes der zuständigen Klinik (Abtei-lung, Institut). 
Einreichungsberechtigt sind Nachwuchskräfte an österreichischen Kliniken, Abteilungen oder Instituten, welche im Besitz der österreichischen

Staatsbürgerschaft und der Mitgliedschaft an der Van-Swieten-Gesellschaft sein müssen. Für die Vergabe des Stipen-diums setzt der Vorstand der Van-SwietenGesellschaft ein vier- bis sechsköpfiges Gremium unter dem Vorsitz des Präsidenten oder eines von ihm bestimmten Vertreters ein. Dieses Gremium ent-scheidet mit Stimmenmehrheit unter Ausschluss des Rechtsweges; bei Stimmengleichheit steht dem Vorsitzenden das Dirimierungs-recht zu.

Nach Abschluss der geförderten Studien ist dem Vorstand der Van-SwietenGesellschaft ein schriftlicher Erfahrungsbericht zu er-statten. Anlässlich der Jahrestagung der Van-Swieten-Gesellschaft hat der Stipendiat über Einladung des Präsidiums ein entsprechen-des Kurzreferat zu halten.

Ende der Einreichungsfrist: 30. Mai 1998 (Datum des Poststempels)

Entsprechende Ansuchen sind an den Sekretär der Van-Swieten-Gesellschaft, p.A. Wr. Med. Akademie, z. Hd. Fr. S. Konstantinou, Alserstrasse 4, A-1090 Wien, zu richten.

232

Mitteilungen aus Österreich 